\title{
QUASI-STATIC HYDRODYNAMIC LIMITS
}

\author{
ANNA DE MASI AND STEFANO OLLA
}

\begin{abstract}
We consider hydrodynamic limits of interacting particles systems with open boundaries, where the exterior parameters change in a time scale slower than the typical relaxation time scale. The limit deterministic profiles evolve quasi-statically. These limits define rigorously the thermodynamic quasi static transformations also for transition between non-equilibrium stationary states. We study first the case of the symmetric simple exclusion, where duality can be used, and then we use relative entropy methods to extend to other models like zero range systems. Finally we consider a chain of anharmonic oscillators in contact with a thermal Langevin bath with a temperature gradient and a slowly varying tension applied to one end.
\end{abstract}

\section{INTRODUCTION}

Quasi-static transformations are defined in thermodynamic literature as those transformations where the external conditions change so slowly that at any moment the system is approximately in equilibrium. They are usually presented as idealization of real thermodynamic transformations [3]. On the other hand they are necessary, in the traditional approach, in order to construct thermodynamic potentials, for example to define thermodynamic entropy from Carnot cycles.

Equilibrium statistical mechanics succesfully describes the equilibrium thermodynamics states from the microscopic dynamical properties of the system, in particular the corresponding stationary probability distributions, given explicitely by the Gibbs measures. Let us

Date: February 26, 2018. File: dmoqs-revised-2.tex.

2000 Mathematics Subject Classification. ...

Key words and phrases. Quasi-Static thermodynamic, hydrodynamic limits, Clausius equality.

We thank Lorenzo Bertini, Claudio Landim, Giovanni Jona Lasinio and Errico Presutti for many very helpful discussions. We thank the kind hospitality of GSSI, AdM thanks also the Institute H. Poincare, where part of this work has been done. The work of S.O. has been partially supported by the European Advanced Grant Macroscopic Laws and Dynamical Systems (MALADY) (ERC AdG 246953). 
consider the simple situation when the internal dynamics of the system has a certain number of conserved quantities (energy, density,...), and the external conditions (temperature of the heat bath, pressure of the external forces,...) select the stationary equilibrium probability distribution for the microscopic dynamics. The fact that, when the microscopic system is large, there are only few of such conserved quantities, and correspondingly few external parameters will determine the equilibrium state, is related to the ergodicity of the microscopic dynamics.

The system can be driven out of equilibrium by changing the exterior parameters. If this is done abruptly, eventually the system will relax to a new equilibrium state corresponding to the new values of temperature, pressure etc.. The typical relaxation time to equilibrium may depend on the size of the microscopic system.

The situation is similar but more complex when the external agents are space inhomogeneous, for example when the system is submitted to gradients of temperature or pressure. In the stationary situation fluxes of the conserved quantities can cross the system. The corresponding probability stationary distributions for the microscopic dynamics are called non-equilibrium stationary states. These are not explicitly computable as in the equilibrium case, but are locally close to the equilibrium ones, when the size of the system is very large.

In the present article we want to obtain, at least for some systems, in a rigorous way the quasi-static thermodynamic transformations from the microscopic dynamics, through a space-time scale limit. In systems of size $N$ with diffusive behavior, where the typical time scale for reaching equilibrium is of order $N^{2}$, we look at the evolution of the conserved quantities at the time scale $N^{2+\alpha} t$, for a $\alpha>0$, and we change the exterior conditions smoothly at this time scale (i.e. very slowly with respect to the typical relaxation time). We obtain that in this time scale, at each instant $t$, the profile of the conserved quantities converges to the deterministic profiles corresponding to the stationary state for the boundary condition given at time $t$. This means that the profiles evolve quasi-statically, following the rules of the thermodynamic quasi-static transformations. Consequently the paradox of the quasi-static transformations disappears when these transformations are understood (also in a mathematically precise way) in a proper macroscopic space-time scale. Remarkably we also obtain quasi-static transformations between non-equilibrium stationary states, that could be the base for constructing a thermodynamics of non-equilibrium.

We consider three different models, in order to illustrate the generality of the idea. 
We consider first the symmetric simple exclusion in an interval of size $N$, i.e. particles performing symmetric random walks with exclusion rule, with density reservoirs at the boundaries. These reservoirs are realized with random creation and annhiliation of particles so that the average density of particles at the boundaries will be respectively $\rho_{-}(t)$ on the left and $\rho_{+}(t)$ on the right. The rate of jumps or of creations/annhiliation is $N^{2+\alpha}, \alpha$ being any positive number. We prove that at every time $t$ the empirical particle's density converges, as $N \rightarrow \infty$, to the linear interpolation between $\rho_{-}(t)$ and $\rho_{+}(t)$, i.e. to the solution of the Laplace equation $\partial_{x x} \rho=0$ with the given boundary conditions. We actually prove the strongest result of the local equilibrium property.

We first prove the above convergence in section 2 by using duality techniques as in [8], where analogous results were proved in the case of $\rho_{ \pm}$fixed constants. With the duality techniques we prove local equilibrium in the strong form of Theorem 2.1 and the same techniques allow to study the limit of the covariance function. In fact we prove in Theorem 2.6 that the covariance behaves at each time $t$ as in the corresponding stationary states, as first computed by Spohn [16].

In Section 3, see Theorem 3.1, we prove a weaker version of the local equilibrium by using entropy methods as in [9] and [11] that are suitable to be used for more general models. In particular the proof of Theorem 3.1 can be adapted to prove the analogous statement for all gradient conservative dynamics with creations/annihilations at the boundaries as the ones studied in [7] in the case of constants $\rho_{ \pm}$.

In Section 4 we consider the zero-range process, where more particles per site are allowed, and jump symmetrically with rate depending on the number of particles in the site they occupy. A chemical potential $\lambda$ characterizes the equilibrium measures, which is the expectation of the jump rate, and in general is a non-linear function of the density. At the boundaries the reservoirs are given again by creations/annihilations of particles corresponding to two possibly different values of $\lambda_{ \pm}(t)$ at time $t$. Jumps and creations/annihilations happen at rate proportional to $N^{2+\alpha}$, and we prove that at each macroscopic time $t$ (in a weak sense) the empirical density of the particles converges to the solution of the non-linear Laplace equation

$$
\partial_{x x} \lambda(\rho(x, t))=0, \quad \lambda(\rho( \pm 1, t))=\lambda_{ \pm}(t) .
$$

Finally, in Section 5, we consider a chain of (unpinned) anharmonic oscillators in contact with a heat bath with a gradient of temperature and subject to a force (tension) $\bar{\tau}(t)$ acting on the last right particle, that changes at the macroscopic time scale. The left end of the chain 
is attached to a point and the heat bath is modeled by Langevin thermostats acting on each particles. The temperatures of the Langevin thermostats may depend on the atom and change slowly form one atom to the neighbor one, giving rise to a smooth macroscopic profile of temperature. When temperature profile $\beta^{-1}(y)$ is constant in space and time at a value $\beta^{-1}$ and tension is constant $\bar{\tau}(t)=\tau$, the stationary (equilibrium) distribution is given by the canonical Gibbs measure with these values of temperaure and tension. In equilibrium the tension $\tau$ is equal to the expectation value of the anharmonic force between particles.

For a non-constant profile of temperature we have a non-equilibrium stationary state where energy flows from hot to cold thermostats. We consider only situations where the profile of temperature does not change in time, and only the applied tension is changing in the macroscopic time scale. Again the dynamics is speeded up in time by $N^{2+\alpha}$. We prove that the empirical strain of volume converge to the solution of the equation

$$
\tau(r(x, t), \beta(x))=\bar{\tau}(t)
$$

where $\tau(r, \beta)$ is the equilibrium tension corresponding to the volume (lenght) $r$ and temperature $\beta^{-1}$, i.e. $\tau=\partial_{r} \mathcal{F}(r, \beta)$, where $\mathcal{F}(r, \beta)$ is the thermodynamic free energy. We have obtained this way the quasistatic isothermal transformation.

The main interest in this last model is that we can define the heat $Q(t)$ as the limit of the the total flux of energy between the system and the thermostats, divided by the number of particles, and it turns out that this is a deterministic function of time that satisfy

$$
U(t)-U(0)=Q(t)+\mathcal{W}(t)
$$

where $U(t)$ is the limit of the internal energy (per particle) and $\mathcal{W}(t)$ is the work done by the exterior force. In the context of the equilibrium case (constant temperature profile), this is the first principle of thermodynamics for isothermal quasi-static transformations. For non-constant temperature profile $Q(t)$ is usually called excess heat. Also this quasistatic transformation satisfies Clausius identity, i.e. the work $\mathcal{W}(t)$ is equal to the time difference of the free energy after properly identifying the macroscopic free energy as integral over space of the equilibrium one.

Quasi-static limits where previously considered on the macroscopic hydrodynamic diffusive equation, rescaling the boundaries parameters after having performed the hydrodynamic limits (cf. [1], [14], [13]), i.e. in a two step approach. The point of this article is to show that 
quasi-static transformations can be obtained in a straightforward way from the microscopic dynamics by considering the proper space-time scaling.

\section{Simple ExClusion With Boundaries}

We consider the exclusion process in $\{0,1\}^{\Lambda_{N}}, \Lambda_{N}:=\{-N, \ldots, N\}$ with reservoirs at the boundaries with density $\rho_{ \pm}(t) \in(0,1)$. We assume that $\rho_{ \pm}(t)$ are Lipschitz continuous. Presumably our results can be generalized to piecewise continuous functions but we did not investigate this case.

Denoting by $\eta(x) \in\{0,1\}$ the occupation number at $x \in \Lambda_{N}$ we define the dynamics via the generator

$$
L_{N, t}=N^{2+\alpha}\left[L_{\text {exc }}+L_{b, t}\right], \quad t \geq 0, \quad \alpha>0
$$

where

$$
L_{\text {exc }} f(\eta)=\frac{1}{2} \sum_{x=-N}^{N-1}\left(f\left(\eta^{(x, x+1)}\right)-f(\eta)\right)=: \frac{1}{2} \sum_{x=-N}^{N-1} \nabla_{x, x+1} f(\eta)
$$

$\eta^{(x, y)}$ is the configuration obtained from $\eta$ by exchanging the occupation numbers at $x$ and $y$, and

$$
L_{b, t} f(\eta)=\sum_{\sigma= \pm} \rho_{\sigma}(t)^{1-\eta(\sigma N)}\left(1-\rho_{\sigma}(t)\right)^{\eta(\sigma N)}\left[f\left(\eta^{\sigma N}\right)-f(\eta)\right]
$$

where $\eta^{x}(x)=1-\eta(x)$, and $\eta^{x}(y)=\eta(y)$ for $x \neq y$.

Theorem 2.1. For any $\alpha>0$ and for any macroscopic time $t>0$ the following holds. For any initial configuration $\eta_{0}$, for any $r \in[-1,1]$ and for any local function $\varphi$

$$
\lim _{N \rightarrow \infty} \mathbb{E}_{\eta_{0}}\left(\theta_{[N r]} \varphi\left(\eta_{t}\right)\right)=<\varphi(\eta)>_{\bar{\rho}(r, t)}=: \hat{\varphi}(\rho(r, t))
$$

where [.] denotes integer part, $\theta$ is the shift operator, $<\cdot>_{\rho}$ is the expectation with respect to the product Bernoulli measure of density $\rho$, and

$$
\bar{\rho}(r, t)=\frac{1}{2}\left[\rho_{+}(t)-\rho_{-}(t)\right] r+\frac{1}{2}\left[\rho_{+}(t)+\rho_{-}(t)\right], \quad r \in[-1,1]
$$

is the quasi-static profile of density at time $t$.

We prove Theorem 2.1 by using the self-duality of the exclusion that can be adapted to our process as we explain in Propositions 2.2 and 2.4 below. The dual process is defined in terms of absorbed stirring walks as explained in Definition 2.1 below. 
In the rest of this section we use the equivalent definition of the dynamics obtained by considering the generator

$$
\hat{L}_{N, s}:=L_{\text {exc }}+L_{b, N^{-2-\alpha} s}
$$

and then studying the process up to times of order $N^{2+\alpha} t, t \geq 0$ being the macroscopic time.

Definition 2.1. The absorbed walkers.

One absorbed random walk is the Markov process in $\Lambda_{N+1}:=[-N-1, N+1] \cap \mathbb{Z}$ that after an exponential time of mean 1 jumps with equal probability on its nearest neighbor sites and when it reaches $\pm(N+1)$ it stays there forever. We denote by $\left\{x_{t}, t \geq 0\right\} a$ trajectory and we denote by $\tau$ the absorbing time:

$$
\tau=\min \left\{\tau_{-(N+1)}, \tau_{N+1}\right\}, \quad \tau_{a}=\inf \left\{t \geq 0: x_{t}=a\right\}
$$

For any positive integer $n$ we also consider the process $\left\{\left(x_{t}^{(1)}, ., x_{t}^{(n)}\right)\right.$, $t \geq 0\}$ of $n$ stirring walks absorbed at $\pm(N+1)$. The stirring process is defined by exchanging independently at rate $\frac{1}{2}$ the "content" in $x$ and $x+1$, so that if $x_{t}^{(i)}=x$ before the exchange then it moves to $x+1$ while if $x_{t}^{(j)}=x+1$ then it moves to $x$ after the exchange (see (2.24) below for the generator of the stirring process). If one disregards labels this is the exclusion process. We denote by $\left\{x_{s}^{(i)}, s \geq 0\right\}, i=1, ., n$ the trajectory of particle $i$ starting from $x_{0}^{(i)}=x_{i}$ and call $\tau_{i}$ its absorption time. We call $\mathcal{L}$ the generator, $\mathcal{P}_{\underline{x}}$ the law and $\mathcal{E}_{\underline{x}}$ the expectation of this process starting from $\underline{x}=\left(x_{1}, ., x_{n}\right)$.

Proposition 2.2. Let $\eta_{0}$ be any initial configuration. Then for all $x \in \Lambda_{N}$

$$
\mathbb{E}_{\eta_{0}}\left[\eta_{t}(x)\right]=\mathcal{E}_{x}\left[\eta_{0}\left(x_{t}\right) \mathbf{1}_{\tau>t}\right]+\mathcal{E}_{x}\left[\mathbf{1}_{\tau \leq t} u_{x_{\tau}}(t-\tau)\right]
$$

where

$$
u_{ \pm(N+1)}(s)=\rho_{ \pm}\left(N^{-(2+\alpha)} s\right)
$$

Below we often write $u_{ \pm}(s)=u_{ \pm(N+1)}(s)$.

Proof. Given $x$ and $t$, a trajectory $\underline{\varkappa}=\left\{x_{s}, s \in[0, t]\right\}$ of the absorbed random walk starting at $x$ and a trajectory $\underline{\eta}=\left\{\eta_{s}, s \in[0, t]\right\}$ starting from $\eta_{0}$, we define for all $s \leq t$

$$
\psi(\underline{\varkappa}, \underline{\eta}, s)= \begin{cases}\eta_{s}\left(x_{t-s}\right), & \text { if } \tau>t-s \\ u_{x_{\tau}}(t-\tau) & \text { if } \tau \leq t-s\end{cases}
$$


Calling $P=\mathcal{P}_{x} \times \mathbb{P}_{\eta_{0}}$ the (product) law of $(\underline{\varkappa}, \underline{\eta})$ and $E$ the expectation with respect to $P$, we observe that for $x \in \Lambda_{N}$

$E(\psi(\underline{\varkappa}, \underline{\eta}, t))=\mathbb{E}_{\eta_{0}}\left(\eta_{t}(x)\right), \quad E(\psi(\underline{\varkappa}, \underline{\eta}, 0))=$ right hand side of (2.8)

Thus to prove (2.8) we show below that $\frac{d}{d s} E[(\psi(\underline{\varkappa}, \underline{\eta}, s))]=0$. We first define for $x \in \Lambda_{N+1}, \eta \in\{0,1\}^{\Lambda_{N}}$ and $a_{ \pm} \in(0,1)$

$$
\varphi_{a_{ \pm}}(x, \eta)= \begin{cases}\eta(x), & \text { if }|x| \leq N \\ a_{ \pm} & \text {if } x= \pm(N+1)\end{cases}
$$

and we will prove that

$$
\frac{d}{d s} E(\psi(\underline{\varkappa}, \underline{\eta}, s))=\left.\left(\frac{d}{d s} E\left(\varphi_{a_{ \pm}}\left(x_{t-s}, \eta_{s}\right)\right)\right)\right|_{a_{ \pm}=u_{ \pm}(t-s)}
$$

Let $0 \leq s<s^{\prime} \leq t$ and call

$$
\begin{aligned}
& D\left(s, s^{\prime}\right)=\psi\left(\underline{\varkappa}, \underline{\eta}, s^{\prime}\right)-\psi(\underline{\varkappa}, \underline{\eta}, s), \\
& \tilde{D}\left(s, s^{\prime}\right)=\varphi_{a_{ \pm}}\left(x_{t-s^{\prime}}, \eta_{s^{\prime}}\right)-\varphi_{a_{ \pm}}\left(x_{t-s}, \eta_{s}\right)
\end{aligned}
$$

First observe that for any choice of $a_{ \pm}$,

$$
D\left(s, s^{\prime}\right) \mathbf{1}_{\tau \leq t-s^{\prime}}=0, \quad \tilde{D}\left(s, s^{\prime}\right) \mathbf{1}_{\tau \leq t-s^{\prime}}=0
$$

If instead $\tau>t-s^{\prime}$ it is important to choose $a_{ \pm}=u_{ \pm}(t-s)$. In fact, since $u_{ \pm}$are Lipschitz continuous functions, we have

$$
\sup _{\sigma \in\left[s, s^{\prime}\right)}\left|\psi(\underline{\varkappa}, \underline{\eta}, \sigma)-\varphi_{u_{ \pm}(t-s)}\left(x_{t-\sigma}, \eta_{\sigma}\right)\right| \mathbf{1}_{\tau>t-s^{\prime}} \leq C\left(s^{\prime}-s\right) \mathbf{1}_{\tau \in\left[t-s^{\prime}, t-s\right]}
$$

Since $P\left(\tau \in\left[t-s^{\prime}, t-s\right]\right) \rightarrow 0$ as $s^{\prime} \downarrow s$, from from (2.15) and (2.16) we get that

$$
\left.\lim _{s^{\prime} \downarrow s} \frac{E\left(D\left(s, s^{\prime}\right)\right)}{s^{\prime}-s}=\left(\lim _{s^{\prime} \downarrow s} \frac{E\left(\tilde{D}\left(s, s^{\prime}\right)\right)}{s^{\prime}-s}\right)\right)\left.\right|_{a_{ \pm}=u_{ \pm}(t-s)}
$$

Thus the Proposition will follows from the fact that the derivative of $E\left(\varphi_{a_{ \pm}}\right)$is 0 . This last derivative is easier to compute because $\varphi_{a_{ \pm}}$is a function of $(x, \eta)$ unlike $\psi$ that is a function of the whole trajectories $(\underline{\varkappa}, \underline{\eta})$. In fact we have

$$
\begin{aligned}
\frac{d}{d s} E & {\left[\varphi_{a_{ \pm}}\left(x_{t-s}, \eta_{s}\right)\right]=-E\left[\mathcal{L} \varphi_{a_{ \pm}}\left(x_{t-s}, \eta_{s}\right)\right]+E\left[\hat{L}_{N, s} \varphi_{a_{ \pm}}\left(x_{t-s}, \eta_{s}\right)\right] } \\
& =-\left[a_{+}-E\left(\eta_{s}(N) \mathbf{1}_{x_{t-s}=N}\right)\right]+\left[u_{+}(t-s)-E\left(\eta_{s}(N) \mathbf{1}_{x_{t-s}=N}\right)\right] \\
& -\left[a_{-}-E\left(\eta_{s}(-N) \mathbf{1}_{x_{t-s}=-N}\right)\right]+\left[u_{-}(t-s)-E\left(\eta_{s}(-N) \mathbf{1}_{x_{t-s}=-N}\right)\right]
\end{aligned}
$$


because the contribution of the generator $L_{\text {exc }}$ cancels with the jumps inside $[-N, N]$ of the random walk. Thus $\frac{d}{d s} E\left[\varphi_{a_{ \pm}}\left(x_{t-s}, \eta_{s}\right)\right]=0$ by putting in (2.17) $a_{ \pm}=u_{ \pm}(t-s)$ in agreement with (2.13).

Corollary 2.3. For any $\alpha>0$, any initial configuration $\eta_{0}$, any $t>0$ and any $r \in[-1,1]$

$$
\lim _{N \rightarrow \infty} \mathbb{E}_{\eta_{0}}\left[\eta_{N^{2+\alpha} t}([N r])\right]=\bar{\rho}(r, t)
$$

Proof. We use (2.8). First observe that for any $x \in \Lambda_{N}$

$$
\mathcal{P}_{x}(\tau>s) \leq c e^{-c^{\prime} s / N^{2}}
$$

thus the first term on the right hand side of (2.8) converges to 0 exponentially. Call

$$
F_{N, x}(s)=\mathcal{P}_{x}\left[\tau_{N+1} \leq s, \tau_{-N-1}>\tau_{N+1}\right]
$$

then by (2.19)

$$
\begin{aligned}
\mid \mathcal{E}_{x}\left[\mathbf{1}_{\tau=\tau_{N+1} \leq N^{2+\alpha} t} u_{N+1}\left(N^{2+\alpha} t-\tau\right)\right] & -\int_{0}^{N^{2+\frac{\alpha}{2}} t} u_{N+1}\left(N^{2+\alpha} t-s\right) d F_{N, x}(s) \mid \\
& \leq c e^{-c^{\prime} N^{\alpha / 2}}
\end{aligned}
$$

Recalling (2.9) we next observe that by Lipschitz continuity

$$
\int_{0}^{N^{2+\alpha / 2} t}\left|u_{N+1}\left(N^{2+\alpha} t-s\right)-\rho_{+}(t)\right| d F_{N, x}(s) \leq c \frac{t N^{2+\alpha / 2}}{N^{2+\alpha}} \leq c \frac{t}{N^{\alpha / 2}}
$$

Finally, by (2.19),

$\left|F_{N, x}\left(N^{2+\alpha / 2} t\right)-\mathcal{P}_{x}\left[\tau_{-(N+1)}>\tau_{N+1}\right]\right| \leq \mathcal{P}_{x}\left[\tau_{N+1}>N^{2+\alpha / 2} t\right] \leq c e^{-c^{\prime} N^{\alpha / 2}}$

and analogously for the term with $u_{-(N+1)}$. Finally observe that

$$
\mathcal{P}_{x}\left(\tau_{N+1} \leq \tau_{-(N+1)}\right)=\frac{1}{2}+\frac{x}{2(N+1)}
$$

We now generalize (2.8) by writing a duality formula for the correlation functions. We thus consider $n>1$ distinct points $x_{1}, . ., x_{n}$ in $\Lambda_{N}$ and recalling Definition 2.1 we prove the following Proposition.

Proposition 2.4. For any positive integer $n$, any $x_{1}, \ldots, x_{n}$ distinct points in $\Lambda_{N}$ and for any $\eta_{0}$ we have that for all $t \geq 0$ (recall (2.9))

$$
\mathbb{E}_{\eta_{0}}\left[\prod_{i=1}^{n} \eta_{t}\left(x_{i}\right)\right]=\mathcal{E}_{\underline{x}}\left[\prod_{i=1}^{n}\left[\mathbf{1}_{\tau_{i}>t} \eta_{0}\left(x_{t}^{(i)}\right)+\mathbf{1}_{\tau_{i} \leq t} u_{x_{\tau_{i}}^{(i)}}\left(t-\tau_{i}\right)\right]\right]
$$


Proof. Denoting by $E=\mathcal{P}_{\underline{x}} \times \mathbb{E}_{\eta_{0}}$ and by $\underline{X}=\left(x_{s}^{(1)}, \ldots, x_{s}^{(n)}, s \in[0, t]\right)$, we prove below that

$$
\frac{d}{d s} E[\Psi(\underline{X}, \underline{\eta}, s)]=0, \quad \Psi(\underline{X}, \underline{\eta}, s)=\prod_{i=1}^{n} \psi\left(\underline{x}^{(i)}, \underline{\eta}, s\right)
$$

The strategy is to adapt the proof given in Proposition 2.2 for $n=1$. Let $\mathcal{F}_{t-s}$ be the sigma-algebra generated by $\left\{\left(x_{s^{\prime}}^{(1)},,, x_{s^{\prime}}^{(n)}\right), s^{\prime} \in[0, t-\right.$ $s]$. Given $\mathcal{F}_{t-s}$ we know $k=$ number of particles absorbed by time $t-s$ and the times of absorption $\tau_{i}<t-s, i=i_{1}, . . i_{k}$. Thus calling

$$
U(t-s):=\prod_{j \in I} u_{x_{\tau_{j}}^{(j)}}\left(t-\tau_{j}\right) \mathbf{1}_{\tau_{j} \leq t-s}, \quad I=\left\{i_{1}, . . i_{k}\right\}
$$

we have that for all $0 \leq s^{\prime}<s \leq t$,

$$
\begin{aligned}
\left.E\left[\Psi\left(\underline{X}, \underline{\eta}, s^{\prime}\right)-\Psi(\underline{X}, \underline{\eta}, s)\right)\right] \\
\quad=E\left[U(t-s) E\left[\prod_{i \notin I} \mathbf{1}_{\tau_{i}>t-s}\left(\psi\left(\underline{x}^{(i)}, \underline{\eta}, s^{\prime}\right)-\psi\left(\underline{x}^{(i)}, \underline{\eta}, s\right)\right) \mid \mathcal{F}_{t-s}\right]\right]
\end{aligned}
$$

Recalling (2.12), as in (2.16) we have that for all $0 \leq s^{\prime}<s \leq t$

$$
\begin{aligned}
& \sup _{\sigma \in\left[s^{\prime}, s\right)} \mid \prod_{i} \mathbf{1}_{\tau_{i}>t-s} \psi\left(\underline{x}^{(i)}, \underline{\eta}, \sigma\right)-\prod_{i} \mathbf{1}_{\tau_{i}>t-s} \varphi_{u_{ \pm}(t-s)}\left(x_{t-\sigma}^{(i)}, \eta, \sigma\right) \mid \\
& \leq C\left(s^{\prime}-s\right) \mathbf{1}_{\exists i: \tau_{i} \in\left[t-s, t-s^{\prime}\right]}
\end{aligned}
$$

Thus as in the case $n=1$, (2.20) is a consequence of

$$
\begin{aligned}
\lim _{s^{\prime} \nearrow s} & \frac{1}{s-s^{\prime}} E\left[U ( t - s ) E \left[\prod_{i \notin I} \mathbf{1}_{\tau_{i}>t-s}\right.\right. \\
& \left.\left.\left(\varphi_{u_{ \pm}(t-s)}\left(x^{(i)}\left(t-s^{\prime}\right), \eta_{s^{\prime}}\right)-\varphi_{u_{ \pm}(t-s)}\left(x^{(i)}(t-s), \eta_{s}\right)\right) \mid \mathcal{F}_{t-s}\right]\right]=0
\end{aligned}
$$

The above equality can be proved by applying the generators similarly to (2.17).

Corollary 2.5. For any initial configuration $\eta_{0}$, for any $t>0$, any $n>1$ and any distinct points $x_{1}, . ., x_{n}$ in $\Lambda_{N}$, calling $t_{N}=N^{2+\alpha} t$

$$
\lim _{N \rightarrow \infty}\left|\mathbb{E}_{\eta_{0}}\left[\prod_{i=1}^{n} \eta_{t_{N}}\left(x_{i}\right)\right]-\prod_{i=1}^{n} \mathbb{E}_{\eta_{0}}\left[\eta_{t_{N}}\left(x_{i}\right)\right]\right|=0
$$

Proof. By (2.20) and (2.19) it is enough to prove that

$$
\lim _{N \rightarrow \infty}\left|D_{\underline{x}, N}\right|=0
$$


where

$$
D_{\underline{x}, N}=\mathcal{E}_{\underline{x}}\left[\prod_{i=1}^{n} \mathbf{1}_{\tau_{i} \leq t_{N}} u_{x_{\tau_{i}}^{(i)}}\left(t_{N}-\tau_{i}\right)\right]-\prod_{i=1}^{n} \mathcal{E}_{x_{i}}\left[\mathbf{1}_{\tau_{i} \leq t_{N}} u_{x_{\tau_{i}}^{(i)}}\left(t_{N}-\tau_{i}\right)\right]
$$

We use the coupling explained in [6] (see also [5]) between $n$ independent random walks and $n$ stirring particles. Both the stirring and the independent particles move in the whole $\mathbb{Z}$. The evolution of the $n$ stirring particles is the process with generator $L^{\text {st }}$ that acts on functions $f$ on $\mathbb{Z}_{\neq}^{n}=\left\{\underline{x} \in \mathbb{Z}^{n}: x_{i} \neq x_{j}\right.$ for all $\left.i \neq j\right\}$ as follows

$$
L^{\mathrm{st}} f(\underline{x})=\frac{1}{2} \sum_{i=1}^{n} \sum_{\sigma= \pm 1}\left[f\left(\underline{x}^{i, \sigma}\right)-f(\underline{x})\right]
$$

with $\underline{x}^{i, \sigma}=\underline{x}+\sigma e_{i}-\sigma e_{j}$ if there is $j \neq i$ such that $x_{j}=x_{i}+\sigma e_{i}$, otherwise $\underline{x}^{i, \bar{\sigma}}=\underline{x}+\sigma e_{i}, e_{i}$ being the unit vector in the $i$ direction. We refer to Section 6.6 of [6] for the definition of the coupling and we denote by $x_{i}^{0}(t)$ and $x_{i}(t)$ the position at time $t$ of the independent and respectively interacting particle with label $i$. The initial positions are the same $\underline{x}^{0}(0)=\underline{x}(0)$ and the law of the coupling is denoted by $Q_{\underline{x}}$ (we denote by $Q_{\underline{x}}$ also the expectation). In Proposition 6.6.3 of [6] it is proved that for any positive $\gamma$ and $k$ there is $c$ so that for all $t>0$

$$
Q_{\underline{x}}\left(\sup _{0<s \leq t}\left|x_{i}(s)-x_{i}^{0}(s)\right| \leq t^{\frac{1}{4}+\gamma}, \forall i=1, . ., n\right) \geq 1-c t^{-k}
$$

We call $\tau_{i}^{0}=\min \left(\tau_{i}^{0,-}, \tau_{i}^{0,+}\right)$ with $\tau_{i}^{0, \pm}$ the first time the independent particle $i$ is at $\pm(N+1), \tau_{i}=\min \left(\tau_{i}^{+}, \tau_{i}^{-}\right)$is the analogue hitting time for the interacting particle $i$. Observe that since the particles are moving by stirring, then the hitting time of interacting particle $i$ is not influenced by the fact that another particle ( say $j$ ) is inside the interval after being outside. Moreover the function inside the expectation in $D_{\underline{x}, N}$ depends only on the hitting times. Thus

$$
\begin{aligned}
& D_{\underline{x}, N}=Q_{\underline{x}}\left(\prod_{i=1}^{n}\left[\mathbf{1}_{\tau_{i} \leq t_{N}} u_{x_{i}\left(\tau_{i}\right)}\left(t_{N}-\tau_{i}\right)\right]\right.
\end{aligned}
$$

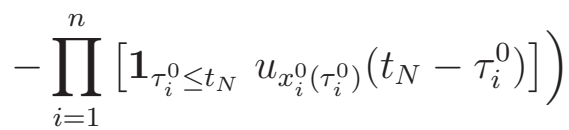

We call $\underline{a}=\left(a_{1}, . ., a_{n}\right), a_{i} \in\{-1,1\}$ the vector that specifies where the stirring particles exit the interval, namely $a_{i}= \pm 1$ if stirring particle $i$ exits in $\pm(N+1)$, thus $\tau_{i}=\tau_{i}^{a_{i}}$. Analogously $\underline{b}=\left(b_{1}, . ., b_{n}\right), b_{i} \in$ $\{-1,1\}$ specifies that $\tau_{i}^{0}=\tau_{i}^{0, b_{i}}$. Given $\underline{a}$ and $\underline{b}$ we denote by $\tau(\underline{a})=$ 
$\left(\tau_{1}^{a_{1}}, . ., \tau_{n}^{a_{n}}\right)$ and by $\tau(\underline{b})=\left(\tau_{1}^{b_{1}}, . ., \tau_{n}^{b_{n}}\right)$. With this notation we have

$$
\begin{gathered}
D_{\underline{x}, N}=\sum_{\underline{a}} \sum_{\underline{b}} Q_{\underline{x}}(\phi(\tau(\underline{a}), \tau(\underline{b}))) \\
\phi(\tau(\underline{a}), \tau(\underline{b}))=\prod_{i=1}^{n}\left[\mathbf{1}_{\tau_{i}=\tau_{i}^{a_{i}} \leq t_{N}} u_{a_{i}}\left(t_{N}-\tau_{i}\right)\right]- \\
\prod_{i=1}^{n}\left[\mathbf{1}_{\tau_{i}^{0}=\tau_{i}^{0, b_{i}} \leq t_{N}} u_{b_{i}}\left(t_{N}-\tau_{i}^{0}\right)\right]
\end{gathered}
$$

Call

$$
A_{N}=\left\{\tau_{i}<N^{2+\alpha / 2}, \tau_{i}^{0}<N^{2+\alpha / 2} \text { for all } i=1, . ., n\right\}
$$

Then by (2.19)

$$
Q_{\underline{x}}(\phi(\tau(\underline{a}), \tau(\underline{b})))=Q_{\underline{x}}\left(\phi(\tau(\underline{a}), \tau(\underline{b})) \mathbf{1}_{A_{N}}\right)+c^{\prime} e^{-c N^{\alpha / 2}}
$$

We now use the Lipschitz continuity of $u_{ \pm}$

$$
\begin{aligned}
& \left|Q_{\underline{x}}\left(\phi(\tau(\underline{a}), \tau(\underline{b})) \mathbf{1}_{A_{N}}\right)-Q_{\underline{x}}\left(\Phi(\tau(\underline{a}), \tau(\underline{b})) \mathbf{1}_{A_{N}}\right)\right| \leq c^{\prime \prime} \frac{1}{N^{a / 2}} \\
& \Phi(\tau(\underline{a}), \tau(\underline{b}))=\prod_{i=1}^{n} u_{a_{i}}\left(t_{N}\right) \mathbf{1}_{\tau_{i}=\tau_{i}^{a_{i}} \leq t_{N}}-\prod_{i=1}^{n} u_{b_{i}}\left(t_{N}\right) \mathbf{1}_{\tau_{i}^{0}=\tau_{i}^{0, b_{i}} \leq t_{N}}
\end{aligned}
$$

Observe now that if $\underline{a}=\underline{b}$ then $\Phi(\tau(\underline{a}), \tau(\underline{b}))=0$, thus

$\mid Q_{\underline{x}}\left(\Phi(\tau(\underline{a}), \tau(\underline{b})) \mathbf{1}_{A_{N}}\right) \leq C \sum_{i=1}^{n} \sum_{b \in\{-1,1\}} Q_{\underline{x}}\left(\mathbf{1}_{\tau_{i}=\tau_{i}^{-b} \leq t_{N}}-\mathbf{1}_{\tau_{i}^{0}=\tau_{i}^{0, b} \leq t_{N}}\right)$

Call

$$
B_{N}=\left\{\left|x_{i}(t)-x_{i}^{0}(t)\right|<N^{\frac{1}{2}+\frac{\alpha}{4}} \text { for all } t \in\left[0, N^{2+\alpha / 2}\right]\right\}
$$

thus by (2.25) for a suitable $\gamma$ and for $k$ sufficiently large

$$
\begin{aligned}
\mid \sum_{b \in\{-1,1\}} Q_{\underline{x}}\left(\mathbf{1}_{\tau_{i}=\tau_{i}^{-b} \leq t_{N}}-\mathbf{1}_{\tau_{i}^{0}=\tau_{i}^{0, b} \leq t_{N}}\right) \\
\quad-Q_{\underline{x}}\left(\mathbf{1}_{B_{N}} \sum_{b \in\{-1,1\}}\left[\mathbf{1}_{\tau_{i}=\tau_{i}^{-b} \leq t_{N}}-\mathbf{1}_{\tau_{i}^{0}=\tau_{i}^{0, b} \leq t_{N}}\right]\right) \mid \leq c N^{-k}
\end{aligned}
$$

Call $X_{N}^{ \pm}=N+1 \mp N^{\frac{1}{2}+\frac{\alpha}{2}}$ and $Y_{N}^{ \pm}=-N-1 \mp N^{\frac{1}{2}+\frac{\alpha}{2}}$. Call $C_{N}=\left\{\right.$ independent particle $i$ reaches $Y_{N}^{+}$and goes to $X_{N}^{-}$before reaching $\left.Y_{N}^{-}\right\}$ $\bigcup\left\{\right.$ independent particle $i$ reaches $X_{N}^{-}$and goes to $Y_{N}^{-}$before reaching $\left.X_{N}^{+}\right\}$. 
Then in $C_{N}^{c}$, the complement set of $C_{N}$, we have that independent and interacting particles $i$ exit from the same side. Thus

$$
Q_{\underline{x}}\left(\mathbf{1}_{C_{N}^{c}} \mathbf{1}_{B_{N}} \sum_{b \in\{-1,1\}} \mathbf{1}_{\tau_{i}=\tau_{i}^{b} \leq t_{N}}\right)=Q_{\underline{x}}\left(\mathbf{1}_{C_{N}^{c}} \mathbf{1}_{B_{N}} \sum_{b \in\{-1,1\}} \mathbf{1}_{\tau_{i}=\tau_{i}^{b} \leq t_{N}} \mathbf{1}_{\tau_{i}^{0}=\tau_{i}^{0}, b \leq t_{N}}\right)
$$

so that

$$
Q_{\underline{x}}\left(\mathbf{1}_{C_{N}^{c}} \mathbf{1}_{B_{N}} \sum_{b \in\{-1,1\}}\left[\mathbf{1}_{\tau_{i}=\tau_{i}^{-b} \leq t_{N}}-\mathbf{1}_{\tau_{i}^{0}=\tau_{i}^{0, b} \leq t_{N}}\right]\right)=0
$$

We are thus left with the trajectories in $C_{N}$ which is an event that involves only the independent particle $i$. Thus

$$
\begin{array}{r}
Q_{\underline{x}}\left(\mathbf{1}_{C_{N}} \mathbf{1}_{B_{N}} \sum_{b \in\{-1,1\}}\left[\mathbf{1}_{\tau_{i}=\tau_{i}^{-b} \leq t_{N}}-\mathbf{1}_{\tau_{i}^{0}=\tau_{i}^{0, b} \leq t_{N}}\right]\right) \leq Q_{\underline{x}}\left(C_{N}\right) \\
\leq \mathcal{P}_{Y_{N}^{+}}^{0}\left(\tau_{X_{N}^{-}}>\tau_{Y_{N}^{-}}\right)+\mathcal{P}_{X_{N}^{-}}^{0}\left(\tau_{Y_{N}^{+}}>\tau_{X_{N}^{+}}\right) \leq c \frac{N^{\frac{1}{2}+\frac{\alpha}{2}}}{2(N+1)}
\end{array}
$$

Proof of Theorem 2.1. Since any cylinder function is a linear combination of functions of the type $\prod_{x} \eta(x)$, (2.4) is a consequence of (2.18) and (2.22).

We conclude this section, by computing at any macroscopic time the covariance of the process. For $x_{1} \neq x_{2} \in \Lambda_{N}$ and $s>0$ we call

$v_{N}\left(x_{1}, x_{2} ; s\right):=\mathbb{E}_{\eta_{0}}\left(\prod_{i=1}^{2}\left[\eta_{s}\left(x_{i}\right)-\rho_{N}\left(x_{i}, s\right)\right]\right), \quad \rho_{N}\left(x_{i}, s\right)=\mathbb{E}_{\eta_{0}}\left(\eta_{s}\left(x_{i}\right)\right)$

We know from Corollary 2.5 that $v_{N}$ converges to 0 as $N \rightarrow \infty$ and in the next Theorem we show that it is of order $N^{-1}$.

Theorem 2.6. For any initial configuration $\eta_{0}$, any $r_{1} \leq r_{2} \in(-1,1)$ and any $t>0$ we have

$$
\lim _{N \rightarrow \infty} N v_{N}\left(\left[N r_{1}\right],\left[N r_{2}\right] ; N^{2+\alpha} t\right)=-\frac{1}{4}\left[\rho_{+}(t)-\rho_{-}(t)\right]^{2} r_{1}\left(1-r_{2}\right)
$$

Furthermore for any $t>s$

$$
\lim _{N \rightarrow \infty} N \mathbb{E}_{\eta_{0}}\left(\left[\eta_{N^{2+\alpha} s}(x)-\rho_{N}\left(x, N^{2+\alpha} s\right)\right]\left[\eta_{N^{2+\alpha} t}(y)-\rho_{N}\left(y, N^{2+\alpha} t\right)\right]\right)=0
$$


Proof. We compute the $t$-derivative of $v_{N}$ :

$$
\begin{aligned}
\frac{d v_{N}}{d t} & =\mathbb{E}_{\eta_{0}}\left(\hat{L}_{N, t}\left[\prod_{i=1}^{2}\left(\eta\left(x_{i}, t\right)-\rho\left(x_{i}, t\right)\right)\right]\right) \\
& -\sum_{i, j=1}^{2} \mathbb{E}_{\eta_{0}}\left(\frac{d \rho_{N}\left(x_{i}, t\right)}{d t}\left[\eta\left(x_{j}, t\right)-\rho\left(x_{j}, t\right)\right] \mathbf{1}_{j \neq i}\right)
\end{aligned}
$$

After easy calculations we get:

$$
\begin{aligned}
\frac{d v_{N}\left(x_{1}, x_{2} ; t\right)}{d t} & =L^{\mathrm{st}, \mathrm{N}} v_{N}\left(x_{1}, x_{2} ; t\right)-\frac{1}{2}\left[\rho_{N}\left(x_{1}, t\right)-\rho_{N}\left(x_{2}, t\right)\right]^{2} \mathbf{1}_{\left|x_{1}-x_{2}\right|=1} \\
& -\frac{1}{2} v_{N}\left(x_{1}, x_{2} ; t\right)\left[\mathbf{1}_{\left|x_{1}\right|=N}+\mathbf{1}_{\left|x_{2}\right|=N}\right]
\end{aligned}
$$

where $v_{N}\left(x_{1}, x_{2} ; t\right)$ is thought of as a function of the positions $x_{1}$ and $x_{2}$ and $L^{\mathrm{st}, \mathrm{N}}$ is the generator defined in (2.24) but with "jumps" outside $\Lambda_{N}$ suppressed. By setting $v_{N}\left(x_{1}, x_{2} ; t\right)=0$ in the set $\bigcup_{i=1}^{2}\left\{\left|x_{i}\right|=N+1\right\}$ we can rewrite (2.33) as an equation for $v_{N}\left(x_{1}, x_{2} ; t\right)$ for $x_{1} \neq x_{2}$ with $\left|x_{i}\right| \leq N+1$ as follows

$$
\begin{aligned}
& \frac{d}{d t} v_{N}\left(x_{1}, x_{2} ; t\right)=L^{\mathrm{st}} v_{N}\left(x_{1}, x_{2} ; t\right)-\frac{1}{2}\left[\rho_{N}\left(x_{1}, t\right)-\rho_{N}\left(x_{2}, t\right)\right]^{2} \mathbf{1}_{\left|x_{1}-x_{2}\right|=1} \\
& v_{N}\left(x_{1}, x_{2} ; t\right)=0 \text { in } \bigcup_{i=1}^{2}\left\{\left|x_{i}\right|=N+1\right\} \text { and when } t=0
\end{aligned}
$$

which is solved by

$$
\begin{gathered}
v_{N}\left(x_{1}, x_{2} ; t\right)=-\frac{1}{2} \int_{0}^{t} E_{x_{1}, x_{2}}^{\mathrm{st}}\left(\left[\rho_{N}\left(x_{1}(s), t-s\right)-\rho_{N}\left(x_{2}(s), t-s\right)\right]^{2}\right. \\
\left.\quad \times \mathbf{1}_{\left|x_{1}(s)-x_{2}(s)\right|=1} \mathbf{1}_{\tau_{1}>s, \tau_{2}>s}\right)
\end{gathered}
$$

where $E_{x, y}^{\mathrm{st}}$ denotes expectation with respect to the process of two stirring particles, labelled 1 and 2 , which start from $x_{1} \neq x_{2} \in \Lambda_{N}$ respectively. $\tau_{1}$ and $\tau_{2}$ are the first time when particle 1 , respectively particle 2 , reaches $\pm(N+1)$. From (2.18) we get that for $t_{N}=N^{2+\alpha} t$

$$
\begin{gathered}
\lim _{N \rightarrow \infty} \sup _{s \leq N^{2+\alpha / 2} t} \sup _{x \in \Lambda_{N}} N^{2} \mid\left[\rho_{N}\left(x, t_{N}-s\right)-\rho_{N}\left(x+1, t_{N}-s\right)\right]^{2} \\
-\frac{1}{2}\left[\rho_{+}(t)-\rho_{-}(t)\right]^{2} \mid=0
\end{gathered}
$$


From (2.19) we get

$$
\begin{gathered}
\mid \int_{0}^{t_{N}} E_{x_{1}, x_{2}}^{\mathrm{st}}\left(\left[\rho_{N}\left(x_{1}(s), t-s\right)-\rho_{N}\left(x_{2}(s), t-s\right)\right]^{2} \mathbf{1}_{\left|x_{1}(s)-x_{2}(s)\right|=1} \mathbf{1}_{\tau_{1}>s, \tau_{2}>s}\right) \\
-\int_{0}^{N^{2+\alpha / 2}} E_{x_{1}, x_{2}}^{\mathrm{st}}\left(\left[\rho_{N}\left(x_{1}(s), t-s\right)-\rho_{N}\left(x_{2}(s), t-s\right)\right]^{2}\right. \\
\left.\quad \times \mathbf{1}_{\left|x_{1}(s)-x_{2}(s)\right|=1} \mathbf{1}_{\tau_{1}>s, \tau_{2}>s}\right) \mid \leq c^{\prime} e^{-c N^{\alpha / 2}}
\end{gathered}
$$

From (2.36) and (2.37),

$$
\begin{aligned}
\lim _{N \rightarrow \infty} & N v_{N}\left(\left[N r_{1}\right],\left[N r_{2}\right] ; N^{2+\alpha} t\right) \\
& =-\frac{1}{4}\left[\rho_{+}(t)-\rho_{-}(t)\right]^{2} \int_{0}^{\infty} E_{r_{1}, r_{2}}\left(\delta_{B(s)}\left(B^{\prime}(s)\right) \mathbf{1}_{\tau>s, \tau^{\prime}>s}\right)
\end{aligned}
$$

$\delta_{b}(x)$ the Dirac delta at $x, B(s)$ and $B^{\prime}(s)$ independent Brownian motions starting from $r_{1}$ and $r_{2}$ and $\tau, \tau^{\prime}$ the hitting times at \pm 1 . As the right hand side of the above expression is identify to the kernel of $(-\Delta)^{-1}$ with Dirichlet boundary conditions, (2.31) easily follows.

To prove (2.32) we observe that

$$
\begin{aligned}
& \mathbb{E}_{\eta_{0}}\left(\left[\eta_{N^{2+\alpha_{s}}}(x)-\rho_{N}\left(x, N^{2+\alpha} s\right)\right]\left[\eta_{N^{2+\alpha} t}(y)-\rho_{N}\left(y, N^{2+\alpha} t\right)\right]\right) \\
& =\mathbb{E}_{\eta_{0}}\left(\left[\eta_{N^{2+\alpha_{s}}}(x)-\rho_{N}\left(x, N^{2+\alpha} s\right)\right] \eta_{N^{2+\alpha} t}(y)\right) \\
& =\mathbb{E}_{\eta_{0}}\left([ \eta _ { N ^ { 2 + \alpha } s } ( x ) - \rho _ { N } ( x , N ^ { 2 + \alpha } s ) ] \mathcal { E } _ { y } \left(\mathbf{1}_{\tau>N^{2+\alpha}(t-s)} \eta_{N^{2+\alpha} s}\left(x\left(N^{2+\alpha}(t-s)\right)\right)\right.\right. \\
& \quad+\mathcal{E}_{y}\left(\mathbf{1}_{\tau \leq N^{2+\alpha}(t-s)} u_{x_{\tau}}(t-\tau)\right) \mathbb{E}_{\eta_{0}}\left(\left[\eta_{N^{2+\alpha} s}(x)-\rho_{N}\left(x, N^{2+\alpha} s\right)\right]\right) \\
& =\mathbb{E}_{\eta_{0}}\left([ \eta _ { N ^ { 2 + \alpha } s } ( x ) - \rho _ { N } ( x , N ^ { 2 + \alpha } s ) ] \mathcal { E } _ { y } \left(\mathbf{1}_{\tau>N^{2+\alpha}(t-s)} \eta_{N^{2+\alpha_{s}}}\left(x\left(N^{2+\alpha}(t-s)\right)\right)\right.\right.
\end{aligned}
$$

that by (2.19) implies (2.32).

As a corollary of Theorem 2.6 one can prove that the fluctuation field converges to a Gaussian field as in the case of constants $\rho_{ \pm}$studied in [12. We do not proof this here.

\section{Simple exclusion: entropy Method}

We prove here the quasi-static limit using the entropy method. This gives a weaker result, but the methods extend to other models where duality cannot be used. We assume here that $\rho_{ \pm}(t)$ are differentiable functions of time with bounded derivative. This can be relaxed to Lipschitz continuity, as assumed in the previous section, but the proof would be more involved.

In the following $\eta_{t}$ is the process generated by (2.1) 
Theorem 3.1. For any $\alpha>0$, any $t>0$, and any local function $\varphi(\eta)$,

$$
\lim _{N \rightarrow \infty} \mathbb{E}_{\eta_{0}}\left(\int_{0}^{t} d s\left|\frac{1}{N} \sum_{x} G\left(\frac{x}{N}\right) \theta_{x} \varphi\left(\eta_{s N^{2+\alpha}}\right)-\int_{0}^{1} G(y) \hat{\varphi}(\bar{\rho}(y, s)) d y\right|\right)=0 .
$$

where $G$ is a continuous test function on $[-1,1]$.

The above theorem is stated for any initial configuration $\eta_{0}$. More generally we can start with any initial distribution, since in the following all we need is that the relative entropies are bounded by $C N$ for some constant $N$, that in this case is automatically satisfied by any probability measure on the configuration space $\{0,1\}^{2 N+1}$.

Consider the empirical distribution of the density at time $t$ :

$$
\xi_{N, t}(G)=\frac{1}{2 N+1} \sum_{x=-N}^{N} G\left(\frac{x}{N}, t\right) \eta_{t}(x)
$$

where $G(y, t)$ is a smooth function on $[-1,1] \times \mathbb{R}_{+}$with compact support in $(-1,1)$. The time evolution is given by

$$
\xi_{N, t}(G)-\xi_{N, 0}(G)=\int_{0}^{t} \xi_{N, s}\left(\left(N^{\alpha} \partial_{y}^{2}+\partial_{s}\right) G\right) d s+O\left(N^{\alpha-1}\right)+M_{N}(t)
$$

where $M_{N}(t)$ is a bounded martingale. By dividing by $N^{\alpha}$, we have immediately that

$$
\lim _{N \rightarrow \infty} \int_{0}^{t} \xi_{N, s}\left(\partial_{y}^{2} G\right) d s=0 .
$$

This implies that every limit point of the empirical distribution $\xi_{N, t}$ is a measure on $[-1,1]$ that satisfies Laplace equation, in a weak sense, with boundary conditions that we will identify in the following.

Corresponding to the quasi-stationary profile $\bar{\rho}(x, t)$ defined in (2.5), we consider the inhomogeneous product measure

$$
\mu_{t}(\eta)=\prod_{x=-N}^{N} \bar{\rho}\left(\frac{x}{N}, t\right)^{\eta(x)}\left[1-\bar{\rho}\left(\frac{x}{N}, t\right)\right]^{1-\eta(x)}
$$

as reference measure at time $t$. The Dirichlet forms associated to the generator are then $\mathfrak{D}_{ \pm N, t, \rho_{ \pm}(t)}(f)$ and $\mathfrak{D}_{e x, t}(f)$ :

$$
\begin{aligned}
& \mathfrak{D}_{x, t, \rho}(f)=\frac{1}{2} \sum_{\eta} \rho^{1-\eta(x)}(1-\rho)^{\eta(x)}\left[\sqrt{f}\left(\eta^{x}\right)-\sqrt{f}(\eta)\right]^{2} \mu_{t}(\eta), \quad x= \pm N \\
& \mathfrak{D}_{e x, t}(f)=\frac{1}{2} \sum_{\eta} \sum_{x=-N}^{N-1}\left(\nabla_{x, x+1} \sqrt{f(\eta)}\right)^{2} \mu_{t}(\eta)
\end{aligned}
$$


Let $f_{N, t}$ be such that the law of $\eta_{t}$ is given by $f_{N, t} \mu_{t}$, the following holds.

Proposition 3.1. There is $C$ so that for all $t$

$$
\int_{0}^{t}\left(\mathfrak{D}_{N, s, \rho_{+}(s)}\left(f_{N, s}\right)+\mathfrak{D}_{-N, s, \rho_{-}(s)}\left(f_{N, s}\right)+\mathfrak{D}_{e x, s}\left(f_{N, s}\right)\right) d s \leq \frac{C t}{N} .
$$

Proof. We have that

$$
\partial_{t}\left(f_{N, t} \mu_{t}\right)=\left(L_{N, t}^{*} f_{N, t}\right) \mu_{t}
$$

where $L_{N, t}^{*}$ is the adjoint with respect to $\mu_{t}(\eta)$ that can be computed as:

$$
\begin{aligned}
& \sum_{\eta} G(\eta)\left(L_{N, t} F\right)(\eta) \mu_{t}(\eta)=\sum_{\eta} F(\eta)\left(L_{N, t} G\right)(\eta) \mu_{t}(\eta) \\
& +N^{2+\alpha} \sum_{\eta} F(\eta) \sum_{x} G\left(\eta^{x, x+1}\right)\left(\mu_{t}\left(\eta^{x, x+1}\right)-\mu_{t}(\eta)\right)
\end{aligned}
$$

Observe that

$$
\left(\mu_{t}\left(\eta^{x, x+1}\right)-\mu_{t}(\eta)\right)=\left[\left(\frac{\bar{\rho}\left(\frac{x+1}{N}, t\right)\left(1-\bar{\rho}\left(\frac{x}{N}, t\right)\right.}{\bar{\rho}\left(\frac{x}{N}, t\right)\left(1-\bar{\rho}\left(\frac{x+1}{N}, t\right)\right.}\right)^{\eta(x)-\eta(x+1)}-1\right] \mu_{t}(\eta)
$$

and

$$
\begin{array}{r}
\left.\left(\frac{\bar{\rho}\left(\frac{x+1}{N}, t\right)\left(1-\bar{\rho}\left(\frac{x}{N}, t\right)\right.}{\bar{\rho}\left(\frac{x}{N}, t\right)\left(1-\bar{\rho}\left(\frac{x+1}{N}, t\right)\right.}\right)^{\eta(x)-\eta(x+1)}-1=\left[1+\frac{1}{N} \frac{\bar{\rho}^{\prime}\left(\frac{x}{N}, t\right)}{\bar{\rho}\left(\frac{x}{N}, t\right)\left(1-\bar{\rho}\left(\frac{x}{N}, t\right)\right.}\right)\right]^{\eta(x)-\eta(x+1)}-1 \\
=\frac{1}{N}[\eta(x)-\eta(x+1)] \frac{\bar{\rho}^{\prime}\left(\frac{x}{N}, t\right)}{\bar{\rho}\left(\frac{x}{N}, t\right)\left(1-\bar{\rho}\left(\frac{x}{N}, t\right)\right)}+O\left(\frac{1}{N^{2}}\right) \\
=: \frac{1}{N}[\eta(x)-\eta(x+1)] B\left(\frac{x}{N}, t\right)+O\left(\frac{1}{N^{2}}\right) .
\end{array}
$$

Denote $H_{N}(t)=\sum_{\eta} f_{N, t}(\eta) \log f_{N, t}(\eta) \mu_{t}(\eta)$

$$
\begin{aligned}
\frac{d}{d t} H_{N}(t) & =\sum_{\eta} \log f_{N, t}(\eta)\left(L_{N, t}^{*} f_{N, t}\right)(\eta) \mu_{t}(\eta)+\sum_{\eta}\left[\partial_{t} f_{N, t}(\eta)\right] \mu_{t}(\eta) \\
& =\sum_{\eta} f_{N, t}(\eta)\left(L_{N, t} \log f_{N, t}\right)(\eta) \mu_{t}(\eta)+\sum_{\eta}\left[\partial_{t} f_{N, t}(\eta)\right] \mu_{t}(\eta)
\end{aligned}
$$

Observe that, since $\frac{d}{d t} \sum_{\eta} f_{N, t}(\eta) \mu_{t}(\eta)=0$, we have

$$
\sum_{\eta}\left[\partial_{t} f_{N, t}(\eta)\right] \mu_{t}(\eta)=-\sum_{\eta} f_{N, t}(\eta)\left[\partial_{t} \mu_{t}(\eta)\right]=O(N)
$$


By the inequality $a \log (b / a) \leq 2 \sqrt{a}(\sqrt{b}-\sqrt{a})$, we have that

$$
f(\eta)\left(L_{N, t} \log f\right)(\eta) \leq 2 \sqrt{f(\eta)}\left(L_{N, t} \sqrt{f}\right)(\eta)
$$

Furthermore

$$
\begin{aligned}
\sum_{\eta} 2 & \sqrt{f_{N, t}(\eta)}\left(L_{N, t} \sqrt{f_{N, t}}\right)(\eta) \mu_{t}(\eta) \\
= & -N^{2+\alpha} \sum_{\eta} \sum_{x=-N}^{N-1} \nabla_{x, x+1} \sqrt{f_{N, t}}(\eta) \nabla_{x, x+1}\left(\sqrt{f_{N, t}} \mu_{t}\right)(\eta) \\
& -N^{2+\alpha}\left(\mathfrak{D}_{N, t, \rho_{+}(t)}\left(f_{N, t}\right)+\mathfrak{D}_{-N, t, \rho_{-}(t)}\left(f_{N, t}\right)\right)
\end{aligned}
$$

and

$$
\begin{array}{r}
\sum_{\eta} \sum_{x=-N}^{N-1} \nabla_{x, x+1} \sqrt{f_{N, t}}(\eta) \nabla_{x, x+1}\left(\sqrt{f_{N, t}} \mu_{t}\right)(\eta)=\sum_{\eta} \sum_{x=-N}^{N-1}\left(\nabla_{x, x+1} \sqrt{f_{N, t}}\right)^{2} \mu_{t} \\
\left.+\sum_{\eta} \sum_{x=-N}^{N-1} \sqrt{f_{N, t}}\left(\eta^{x, x+1}\right) \nabla_{x, x+1}\left(\sqrt{f_{N, t}}\right)(\eta) \nabla_{x, x+1} \mu_{t}(\eta)\right) \\
=\sum_{\eta} \sum_{x=-N}^{N-1}\left(\nabla_{x, x+1} \sqrt{f_{N, t}}\right)^{2} \mu_{t} \\
+\frac{1}{N} \sum_{\eta} \sum_{x=-N}^{N-1} \sqrt{f_{N, t}}\left(\eta^{x, x+1}\right) \nabla_{x, x+1}\left(\sqrt{f_{N, t}}\right)[\eta(x)-\eta(x+1)] B\left(\frac{x}{N}, t\right) \mu_{t}+O\left(N^{-1}\right) \\
=2 \mathfrak{D}_{e x, t}\left(f_{N, t}\right)+\frac{1}{N} \tilde{B}_{N}(t)+O\left(N^{-1}\right)
\end{array}
$$

with

$$
\tilde{B}_{N}(t)=\sum_{\eta} \sum_{x=-N}^{N-1} \sqrt{f_{N, t}}\left(\eta^{x, x+1}\right) \nabla_{x, x+1}\left(\sqrt{f_{N, t}}\right)(\eta(x)-\eta(x+1)) B\left(\frac{x}{N}, t\right) \mu_{t}
$$

By an elementary inequality we have:

$$
\begin{aligned}
&\left|\tilde{B}_{N}(t)\right| \leq \frac{N}{2} \mathfrak{D}_{e x, t}\left(f_{N, t}\right)+\frac{2}{2 N} \sum_{\eta} \sum_{x=-N}^{N-1} f_{N, t}\left(\eta^{x, x+1}\right)(\eta(x)-\eta(x+1))^{2} B\left(\frac{x}{N}, t\right)^{2} \mu_{t} \\
& \leq \frac{N}{2} \mathfrak{D}_{e x, t}\left(f_{N, t}\right)+\frac{2}{2 N} \sum_{x=-N}^{N-1} B\left(\frac{x}{N}, t\right)^{2} \sum_{\eta} f_{N, t}(\eta) \mu_{t}\left(\eta^{x, x+1}\right) \\
&=\frac{N}{2} \mathfrak{D}_{e x, t}\left(f_{N, t}\right)+\frac{2}{2 N} \sum_{x=-N}^{N-1} B\left(\frac{x}{N}, t\right)^{2}\left(1+\sum_{\eta} f_{N, t}(\eta) \nabla_{x, x+1} \mu_{t}\right)
\end{aligned}
$$


and since $B(x, t) \leq \frac{1}{4}\left\|\bar{\rho}^{\prime}\right\|_{\infty}$, iterating on the bound for $\nabla_{x, x+1} \mu_{t}$, we obtain

$$
\left|\tilde{B}_{N}(t)\right| \leq \frac{N}{a} \mathfrak{D}_{e x, t}\left(f_{N, t}\right)+C a
$$

All together we have

$$
\begin{gathered}
H_{N}(t)-H_{N}(0)=-N^{2+\alpha} \int_{0}^{t}\left(\mathfrak{D}_{N, s, \rho_{+}(s)}\left(f_{N, s}\right)+\mathfrak{D}_{-N, s, \rho_{-}(s)}\left(f_{N, s}\right)+\mathfrak{D}_{e x, s}\left(f_{N, s}\right)\right) d s \\
\quad-N^{1+\alpha} \int_{0}^{t} \tilde{B}_{N}(s) d s+O\left(N^{1+\alpha}\right) t \\
\leq-N^{2+\alpha} \int_{0}^{t}\left(\mathfrak{D}_{N, \rho_{+}(s)}\left(f_{N, s}\right)+\mathfrak{D}_{-N, s, \rho_{-}(s)}\left(f_{N, s}\right)+\mathfrak{D}_{e x, s}\left(f_{N, s}\right)\right) d s \\
\quad+\frac{N^{2+\alpha}}{2} \int_{0}^{t} \mathfrak{D}_{e x, s}\left(f_{N, s}\right) d s+N^{1+\alpha} 2 C t+O\left(N^{1+\alpha}\right) t \\
\int_{0}^{t}\left(\mathfrak{D}_{N, \rho_{+}(s)}\left(f_{N, s}\right)+\mathfrak{D}_{-N, \rho_{-}(s)}\left(f_{N, s}\right)+\frac{1}{2} \mathfrak{D}_{e x}\left(f_{N, s}\right)\right) d s \\
\leq \frac{H_{N}(0)+N^{1+\alpha} 2 C t+O\left(N^{1+\alpha}\right) t}{N^{2+\alpha}} \leq \frac{C^{\prime \prime}}{N^{1+\alpha}}+\frac{C^{\prime \prime \prime} t}{N} .
\end{gathered}
$$

Proof of Theorem [3.1. Consider for any point $y \in[-1+k / N, 1-k / N]$ the set $\Lambda_{y}$ of $2 k+1$ integers defined by $\Lambda_{y}=\{[N y]-k, \ldots,[N y]+k\}$, and define $\left.f_{N, t}\right|_{\Lambda_{y}}$ the marginal of $f_{N, t}$ on $\Lambda_{y}$. It follows from (3.7) that for $f_{t, y}=\left.\lim _{N \rightarrow \infty} f_{N, t}\right|_{\Lambda_{y}}$

$$
\mathfrak{D}_{e x, k, t, y}\left(f_{t, y}\right):=\sum_{\eta \in\{0,1\}^{2 k+1}} \sum_{x=-k}^{k-1}\left(\sqrt{f_{t, y}}\left(\eta^{x, x+1}\right)-\sqrt{f_{t, y}}(\eta)\right)^{2} \mu_{\rho(y, t)}^{k}=0
$$

(where $\mu_{\rho(y, t)}^{k}$ is the Bernoulli measure on $\{0,1\}^{2 k+1}$ with density $\rho(y, t)$ ). This implies that $f_{t, y}\left(\eta_{-k}, \ldots, \eta_{k}\right)$ is symmetric for exchanges. Furthermore, considering the boundary blocks $\{-N, \ldots,-N+2 k\}$ and $\{N-2 k, \ldots, N\}$ we obtain respectively

$$
\mathfrak{D}_{N, t, \rho_{+}(t)}\left(f_{t,-1}\right)=0, \quad \mathfrak{D}_{-N, t, \rho_{-}(t)}\left(f_{t, 1}\right)=0
$$

that implies $f_{t, 1}$ and $f_{t,-1}$ are constant, and since they are probability densities with respect to $\mu_{\rho_{+}(t)}^{k}$ and $\mu_{\rho_{-}(t)}^{k}$, they are equal to 1 in those boundary blocks. 
From the above argument we obtain that

$$
\begin{aligned}
& \lim _{k \rightarrow \infty} \lim _{N \rightarrow \infty} \int_{0}^{t} d s \int_{-1+k / N}^{1-k / N} d y \sum_{\eta} f_{N, s}(\eta) \mu_{s}(\eta) \\
& \left|\frac{1}{2 k} \sum_{|x-N y| \leq k} \theta_{x} \varphi\left(\eta_{s}\right)-\hat{\varphi}\left(\frac{1}{2 k} \sum_{|x-N y| \leq k} \eta(x)\right)\right|=0 .
\end{aligned}
$$

Next we extend this statement to macroscopic blocks. Consider now, for any $y, y^{\prime} \in[-1+k / N, 1-k / N]$ the two blocks $\Lambda_{y}$ and $\Lambda_{y^{\prime}}$, and let $f_{N, t, y, y^{\prime}}(\eta, \tilde{\eta})$ the corresponding joint marginal. Define, for functions on two separate blocks $f\left(\eta_{-k}, \ldots, \eta_{k} ; \tilde{\eta}_{-k}, \ldots, \tilde{\eta}_{k}\right)$, the Dirichlet form corresponding to the exchange of the occupation of the centers of the box:

$$
\mathfrak{D}_{\mathfrak{o}}(f)=\sum_{\eta, \tilde{\eta}}\left(\sqrt{f}\left(T_{0}(\eta, \tilde{\eta})\right)-\sqrt{f}(\eta, \tilde{\eta})\right)^{2} \mu_{\bar{\rho}(t, y)}^{k}(\eta) \mu_{\bar{\rho}\left(t, y^{\prime}\right)}^{k}(\tilde{\eta})
$$

where $T_{0}$ is the exchange of $\eta(0)$ with $\tilde{\eta}(0)$.

By the same telescoping argument used in [11], we have that $f_{t, y, y^{\prime}}(\eta, \tilde{\eta})=$ $\lim f_{N, t, y, y^{\prime}}(\eta, \tilde{\eta})$, satisfies

$$
\mathfrak{D}_{\mathfrak{o}}\left(f_{t, y, y^{\prime}}\right) \leq C\left|y-y^{\prime}\right|^{2}
$$

It follows that (see [10] for standard details):

$$
\begin{aligned}
\lim _{\epsilon \rightarrow 0} \lim _{k \rightarrow \infty} \lim _{N \rightarrow \infty} \int_{0}^{t} d s \int_{-1+k / N}^{1-k / N} d y \sum_{\eta} f_{N, s}(\eta) \mu_{s}(\eta) \\
\left(\frac{1}{2 k+1} \sum_{x=[N y]-k}^{[N y]+k} \eta(x)-\frac{1}{2 N \epsilon} \sum_{x=[N(y-\epsilon)]}^{[N(y+\epsilon)]} \eta(x)\right)^{2}=0
\end{aligned}
$$

In fact we already know that

$$
\lim _{k \rightarrow \infty} \lim _{N \rightarrow \infty} \int_{0}^{t} d s \sum_{\eta}\left(\frac{1}{2 k+1} \sum_{x=-N}^{-N+2 k} \eta(x)-\rho_{-}(s)\right)^{2} f_{N, s}(\eta) \mu_{s}(\eta)=0
$$

and similarly for the block $[N-2 k, N]$. By (3.10) we have

$$
\lim _{\epsilon \rightarrow 0} \lim _{N \rightarrow \infty} \int_{0}^{t} d s \sum_{\eta}\left(\frac{1}{2 N \epsilon} \sum_{x=-N}^{-N(1+2 \epsilon)} \eta(x)-\rho_{-}(s)\right)^{2} f_{N, s}(\eta) \mu_{s}(\eta)=0
$$


This implies, with (3.4), that the limit of $\frac{1}{2 N \epsilon} \sum_{x=[N(y-\epsilon)]}^{[N(y+\epsilon)]} \eta(x)$ as $N \rightarrow$ $\infty$ and $\epsilon \rightarrow 0$ converges strongly to the solution of the Laplace equation with this boundary condition given by $\rho_{ \pm}(t)$, i.e. $\bar{\rho}(y, t)$.

\section{ZERO RANGE}

The results of the previous section extend quite straightforwardly to any gradient conservative dynamic. As an example, let us consider the zero range process, whose generator is given by

$$
\begin{aligned}
L_{N, t}^{0 \mathrm{r}} f(\eta)=\frac{N^{2+\alpha}}{2}\{ & \sum_{x=-N}^{N} g(\eta(z)) \sum_{\sigma= \pm 1}\left[f\left(\eta^{x, x+\sigma}\right)-f(\eta)\right] \\
& \left.+\sum_{\sigma= \pm} \lambda_{\sigma}(t)\left[f\left(\eta^{\sigma N,+}\right)-f(\eta)\right]\right\}
\end{aligned}
$$

where $\eta^{x, y}$ is the configuration $\eta$ with a particle moved from $x$ to $y$, for $x, y=-N, \ldots, N$. For $\eta^{N, N+1}$ and $\eta^{-N,-N-1}$ we have destroyed a particle in the correspondig site, while $\eta^{x,+}$ means we have addeed a particle in the site $x=N,-N$. We assume that the rate function $g: \mathbb{N} \rightarrow \mathbb{R}_{+}$such that $g(0)=0, g(k)>0$ for $k>0$, and $\sup _{k} \mid g(k+$ $1)-g(k) \mid<+\infty$. As before we assume that $\lambda_{ \pm}(t)$ are differentiable functions of time with bounded derivative.

For any $\lambda>0$ consider the measure $\mu_{\lambda}$ on the non negative integer

$$
\mu_{\lambda}(k)=\frac{\lambda^{k}}{g(k) !} \frac{1}{Z(\lambda)}, \quad g(k) !=g(1) \ldots g(k), g(0) !=1 .
$$

where $Z(\lambda)$ is the normalization constant.

Let $\bar{\lambda}(r, t), r \in[-1,1]$ be the linear interpolation of $\lambda_{ \pm}(t)$ defined as in (2.5).

Consider as reference measure the inhomogeneous product

$$
\mu_{t}^{N}(\eta)=\prod_{x=-N}^{N} \mu_{\bar{\lambda}\left(\frac{x}{N}, t\right)}(\eta(x))
$$

Observe that for all $N, t$ and all local function $\varphi$ :

$$
\sum_{\eta} L_{N, t}^{0 \mathrm{r}} \varphi(\eta) \mu_{t}^{N}(\eta)=0
$$

For constant $\lambda$, we denote by $\mu_{\lambda}$ the corresponding homogeneoous product measure.

Assume that the initial configuration $\eta_{0}$ is randomly distributed by a probability measure with density $f_{N, 0}$ with respect to $\mu_{0}^{N}(\eta)$ and such 
that the relative entropy $\sum_{\eta} f_{N, 0}(\eta) \log f_{N, 0}(\eta) \mu_{0}^{N}(\eta) \leq C N$ for some constant $C>0$.

Theorem 4.1. For any local function $\varphi$, denoting $\hat{\varphi}(\lambda)=<\varphi>_{\lambda}$, the average of $\varphi$ with respect to $\mu_{\lambda}$ :

$$
\lim _{N \rightarrow \infty} \mathbb{E}\left(\int_{0}^{t} d s\left|\frac{1}{N} \sum_{x} G\left(\frac{x}{N}\right) \theta_{x} \varphi\left(\eta_{s}\right)-\int_{0}^{1} G(y) \hat{\varphi}(\bar{\lambda}(y, s)) d y\right|\right)=0 .
$$

where $G$ is a measurable bounded test function and $\theta_{i}$ is the space shift by $i$, well defined for $N$ large enough.

We omit the proof of Theorem 4.1 being very similar to the one of Theorem 3.1 for simple exclusion.

As observed in [4] in the case of $\lambda_{ \pm}$constants, (4.3) straightforwardly yields (4.4).

\section{DAmped ANHARMONiC CHAin IN TEMPERATURE GRADIENT}

We consider a chain of $N$ coupled oscillators in one dimension. Each particle has the same mass, equal to one. The configuration in the phase space is described by $\eta=\left\{q_{x}, p_{x}, x=1, \ldots, N\right\} \in \mathbb{R}^{2 N}$. The interaction between two particles $x$ and $x-1$ is described by the potential energy $V\left(q_{x}-q_{x-1}\right)$ of an anharmonic spring. The chain is attached on the left to a fixed point, so we set $q_{0}(t)=0, p_{0}(t)=0$. We call $\left\{r_{x}=q_{x}-q_{x-1}, x=1, \ldots, N\right\}$ the interparticle distance. We assume $\mathrm{V}$ to be a positive smooth function, and that there exists a constant $C>0$ such that:

$$
\lim _{|r| \rightarrow \infty} \frac{V(r)}{|r|}=\infty, \quad \lim _{|r| \rightarrow \infty} V^{\prime \prime}(r) \leq C<\infty
$$

Energy is defined by the following Hamiltonian:

$$
\mathcal{H}:=\sum_{x=1}^{N}\left(\frac{p_{x}^{2}}{2}+V\left(r_{x}\right)\right)
$$

The particle dynamics is subject to an interaction with an environment given by Langevin heat bath at different temperatures $\beta_{x}^{-1}$. We choose $\beta_{x}$ as slowly varying on a macroscopic scale, i.e. $\beta_{x}=\beta(x / N)$ for a given smooth strictly positive function $\beta(y), y \in[0,1]$. 
The equations of motion are given by

$$
\left\{\begin{aligned}
d r_{x}(t)= & N^{2+\alpha}\left(p_{x}(t)-p_{x-1}(t)\right) d t \\
d p_{x}(t)= & N^{2+\alpha}\left(V^{\prime}\left(r_{x+1}(t)\right)-V^{\prime}\left(r_{x}(t)\right)\right) d t \\
& -N^{2+\alpha} \gamma p_{x}(t) d t+N^{1+\alpha / 2} \sqrt{\frac{2 \gamma}{\beta_{x}}} d w_{x}(t), \quad x=1, \ldots, N-1 \\
d p_{N}(t)= & N^{2+\alpha}\left(\bar{\tau}(t)-V^{\prime}\left(r_{N}(t)\right)\right) d t-N^{2+\alpha} \gamma p_{N}(t) d t \\
& +N^{1+\alpha / 2} \sqrt{\frac{2 \gamma}{\beta_{N}}} d w_{N}(t) .
\end{aligned}\right.
$$

Here $\left\{w_{x}(t)\right\}_{x}$ are $N$-independent Wiener processes, $\gamma>0$ is the coupling parameter with the Langevin thermostats. The force $\bar{\tau}(t)$ is assumed to be a smooth function of the macroscopic time $t$.

The generator of the process is given by

$$
\mathcal{L}_{n}^{\bar{\tau}(t)}:=N^{2+\alpha}\left(\mathcal{A}_{N}^{\bar{\tau}(t)}+\gamma \mathcal{S}_{N}\right)
$$

where $\mathcal{A}_{N}^{\bar{\tau}}$ is the Liouville generator

$$
A_{N}^{\bar{\tau}}=\sum_{x=1}^{N}\left(p_{x}-p_{x-1}\right) \partial_{r_{N}}+\sum_{x=1}^{N-1}\left(V^{\prime}\left(r_{x+1}\right)-V^{\prime}\left(r_{x}\right)\right) \partial_{p_{x}}+\left(\bar{\tau}-V^{\prime}\left(r_{N}\right)\right) \partial_{p_{N}}
$$

while $\mathcal{S}_{N}$ is the operator

$$
\mathcal{S}_{N}=\sum_{x=1}^{N}\left(\beta_{x}^{-1} \partial_{p_{x}}^{2}-p_{x} \partial_{p_{x}}\right)
$$

An equivalent dynamics is given by a different modelling of the heat bath: particle $x$ undergoes stochastic elastic collisions with particles of the environment at temperature $\beta_{x}^{-1}$, i.e. at independent exponentially distributed times of intensity $\gamma N^{2+\alpha}$ particle $x$ changes its velocity to a new random velocity normally distributed with variance $\beta_{x}^{-1}$. The evolution equations are given by:

$$
\left\{\begin{aligned}
d r_{x}(t)= & N^{2+\alpha}\left(p_{x}(t)-p_{x-1}(t)\right) d t \\
d p_{x}(t)= & N^{2+\alpha}\left(V^{\prime}\left(r_{x+1}(t)\right)-V^{\prime}\left(r_{x}(t)\right)\right) d t \\
& +\left(\tilde{p}_{x, \mathcal{N}_{x}\left(\gamma N^{2+\alpha} t\right)}-p_{x}\left(t^{-}\right)\right) d \mathcal{N}_{x}\left(\gamma N^{2+\alpha} t\right), \quad x=1, . ., N-1 \\
d p_{N}(t)= & N^{2+\alpha}\left(\bar{\tau}(t)-V^{\prime}\left(r_{N}\right)\right) d t \\
& +\left(\tilde{p}_{N, \mathcal{N}_{N}\left(\gamma N^{2+\alpha} t\right)}-p_{N}\left(t^{-}\right)\right) d \mathcal{N}_{N}\left(\gamma N^{2+\alpha} t\right)
\end{aligned}\right.
$$

where $\tilde{p}_{x, k}$ are independent gaussian variables on mean zero and variance $\beta_{x}^{-1}$, and $\left\{\mathcal{N}_{x}(t), x=1, \ldots, N\right\}$ are independent Poisson processes of intensity 1. 
For $\bar{\tau}(t)=\tau$ constant, and $\beta_{x}=\beta$ homogeneous, the system has a unique invariant measure given by a product of invariant Gibbs measures $\mu_{\tau, \beta}^{N}$ :

$$
d \mu_{\tau, \beta}^{N}=\prod_{x=1}^{N} e^{-\beta\left(\mathcal{E}_{x}-\tau r_{x}\right)-\mathcal{G}_{\tau, \beta}} d r_{x} d p_{x}
$$

where $\mathcal{E}_{x}$ is the energy of the particle $x$ :

$$
\mathcal{E}_{x}=\frac{p_{x}^{2}}{2}+V\left(r_{x}\right)
$$

The function $\mathcal{G}_{\tau, \beta}$ is the Gibbs potential defined as:

$$
\mathcal{G}_{\tau, \beta}=\log \left[\sqrt{2 \pi \beta^{-1}} \int e^{-\beta(V(r)-\tau r)} d r\right] .
$$

The free energy of the equilibrium state $(r, \beta)$ is given by the Legendre transform of $-\beta^{-1} \mathcal{G}_{\tau, \beta}$ :

$$
\mathcal{F}_{r, \beta}=\sup _{\tau}\left\{\tau r+\beta^{-1} \mathcal{G}_{\tau, \beta}\right\}
$$

The corresponding convex conjugate variables are the lenght

$$
\mathfrak{r}(\tau, \beta)=\beta^{-1} \partial_{\tau} \mathcal{G}_{\tau, \beta}
$$

and the tension

$$
\boldsymbol{\tau}(r, \beta)=\partial_{r} \mathcal{F}_{r, \beta}
$$

Observe that

$$
\mathbb{E}_{\mu_{\tau, \beta}^{N}}[r]=\mathfrak{r}, \quad \mathbb{E}_{\mu_{\tau, \beta}^{N}}\left[V^{\prime}(r)\right]=\boldsymbol{\tau}
$$

Let $\nu_{\beta}^{N}$ the inhomogeneous Gibbs measure

$$
d \nu_{\beta .}^{N}=\prod_{x=1}^{N} \frac{e^{-\beta_{x} \mathcal{E}_{x}}}{Z_{\beta_{x}}}
$$

Observe that this is not the stationary measure for this dynamics for $\bar{\tau}=0$.

Also we will use the thermodynamic entropy defined as

$$
S(u, r)=\inf _{\beta>0}\{-\beta u-\beta \mathcal{F}(r, \beta)\} .
$$

Let $f_{t}^{N}$ the density, with respect to $\nu_{\beta}^{N}$, of the probability distribution of the system at time t, i.e. the solution of

$$
\partial_{t} f_{t}^{N}=\mathcal{L}_{N}^{\bar{\tau}(t), *} f_{t}^{N}
$$


where $\mathcal{L}_{N}^{\bar{\tau}(t), *}$ is the adjoint of $\mathcal{L}_{N}^{\bar{\tau}(t)}$ with respect to $\nu_{\beta}^{N}$, i.e. explicitely

$$
\begin{aligned}
\left(\mathcal{L}_{N}^{\bar{\tau}(t)}\right)^{*}=-N^{2+\alpha} \mathcal{A}_{N}^{\tau(t)}- & N^{2+\alpha} \sum_{x=1}^{N-1}\left(\beta_{x+1}-\beta_{x}\right) p_{x} V^{\prime}\left(r_{x+1}\right) \\
& +N^{2+\alpha} \beta(1) p_{x} \bar{\tau}(t)+N^{2+\alpha} \gamma \mathcal{S}_{N} .
\end{aligned}
$$

Define the relative entropy of $f_{t}^{N} d \nu_{\beta}^{N}$. with respect to $d \nu_{\beta}^{N}$ :

$$
H_{N}(t)=\int f_{t}^{N} \log f_{t}^{N} d \nu_{\beta}^{N}
$$

We assume that the initial density $f_{0}^{N}$ satisfy the bound

$$
H_{N}(0) \leq C N
$$

We also need some regularity of $f_{0}^{N}$ : define the hypercoercive Fisher information functional:

$$
I_{N}(t)=\sum_{x=1}^{N-1} \beta_{x}^{-1} \int \frac{\left(\partial_{p_{x}} f_{t}^{N}+\partial_{q_{x}} f_{t}^{N}\right)^{2}}{f_{t}^{N}} d \nu_{\beta}
$$

where $\partial_{q_{x}}=\partial_{r_{x}}-\partial_{r_{x+1}}, x=1, \ldots, N-1$, and $\nu_{\beta}:=\nu_{\beta}^{N}$. We assume that

$$
I_{N}(0) \leq K_{N}
$$

with $K_{N}$ growing less than exponential in $N$.

Consider a local function $\varphi(\eta)$ such that

$$
|\varphi(\eta)| \leq C \sum_{x \in \Lambda_{\varphi}}\left(p_{x}^{2}+V\left(r_{x}\right)\right)^{\delta}, \quad \delta<1
$$

where $\Lambda_{\phi}$ is the local support of $\varphi$. Let $\theta_{i} \varphi$ be the shifted function (well defined for large enough $N$ ). Denoting $\hat{\varphi}(\tau, \beta)=\langle\varphi\rangle_{\tau, \beta}$ the expectation with respect to $d \mu_{\tau \beta}^{N}$ defined by (5.8)

\section{Proposition 5.1.}

$\lim _{N \rightarrow \infty} \mathbb{E}^{N}\left(\int_{0}^{t} d s\left|\frac{1}{N} \sum_{x} G\left(\frac{x}{N}\right) \theta_{x} \varphi\left(\eta_{s}\right)-\int_{0}^{1} G(y) \hat{\varphi}(\bar{\tau}(s), \beta(y)) d y\right|\right)=0$.

The proof uses similar ideas as in the previous cases, plus results contained in [13]. In particular from same argument used in [13] follows that the empirical distribution for the $r$ 's converge to the solution of

$$
\int_{0}^{t} d s\left[\int_{0}^{1} \partial_{y}^{2} G(y, s) \boldsymbol{\tau}(r(y, s), \beta(y)) d y-\partial_{y} G(1, s) \bar{\tau}(s)\right]=0
$$

for any smooth function $G(y, t)$ on $[0,1]$ such that $G(1)=0$ and $G^{\prime}(0)=$ 0 . 
5.1. Some "thermodynamic" consequences. We illustrate here how the above limit realizes the so-called quasi-static isothermal trasformation of the thermodynamics. When a temperature gradient is present, 'isothermal' should be intended that the temperature gradient does not change in time. In this case these are quasistatic transformations between non-equilibrium stationary states.

When performing the usual diffusive scaling, as done in [14] for constant temperatures and in [13] for the temperature gradient case, the results below are obtained in a two step limit, first the hydrodunamic diffusive limit, then a quasi-static limit, see details in [14, 13.

5.1.1. Excess Heat. The (normalized) total internal energy of the system is defined by

$$
U_{N}:=\frac{1}{N} \sum_{x=1}^{N}\left(\frac{p_{x}^{2}}{2}+V\left(r_{x}\right)\right)
$$

then internal energy evolves as:

$$
U_{N}(t)-U_{N}(0)=\mathcal{W}_{N}(t)+Q_{N}(t)
$$

where

$$
\mathcal{W}_{N}(t)=N^{1+\alpha} \int_{0}^{t} \bar{\tau}(s) p_{N}(s) d s=\int_{0}^{t} \bar{\tau}(s) \frac{d q_{N}(s)}{n}
$$

is the (normalized) work done by the force $\bar{\tau}(s)$ up to time $t$, while

$$
\begin{aligned}
Q_{N}(t)= & \gamma N^{1+\alpha} \sum_{x=1}^{N} \int_{0}^{t} d s\left(p_{x}^{2}(s)-\beta_{x}^{-1}\right) \\
& +N^{\alpha / 2} \sum_{x=1}^{N} \sqrt{2 \gamma \beta_{x}^{-1}} \int_{0}^{t} p_{x}(s) d w_{x}(s) .
\end{aligned}
$$

is the total flux of energy between the system and the heat bath (divided by $N)$. As a consequence of theorem 5.1 we have that

$$
\lim _{N \rightarrow \infty} \mathcal{W}_{N}(t)=\int_{0}^{t} \bar{\tau}(s) d \mathcal{L}(s):=\mathcal{W}(t)
$$

where $\mathcal{L}(t)=\int_{0}^{1} r(y, t) d y$, the total macroscopic length at time $t$. While for the energy difference we expect that

$$
\lim _{n \rightarrow \infty}\left(U_{N}(t)-U_{N}(0)\right)=\int_{0}^{1}[u(\bar{\tau}(t), \beta(y))-u(\bar{\tau}(0), \beta(y))] d y
$$

where $u(\tau, \beta)$ is the average energy for $\mu_{\beta, \tau}$, i.e.

$$
u(\tau, \beta)=\int \mathcal{E}_{1} d \mu_{\tau, \beta}^{1}=\frac{1}{2 \beta}+\int V(r) e^{-\beta(V(r)-\tau r)-\tilde{\mathcal{G}}(\tau, \beta)} d r
$$


with $\tilde{\mathcal{G}}(\tau, \beta)=\log \int e^{-\beta(V(r)-\tau r)} d r$. Unfortunately for lack of uniform integrability for the energy distribution, we do not have a rigorous proof of (5.28), since energy correspond to a value $\delta=2$ in (5.23). Assuming that the local equilibrium established in 5.1 extends to quadratic growing functions, then we have obtained

$$
Q_{N}(t) \underset{N \rightarrow \infty}{\longrightarrow} Q(t)
$$

where $Q(t)$ is deterministic and satisfy the relation

$$
Q(t)=\int_{0}^{1}[u(\bar{\tau}(t), \beta(y))-u(\bar{\tau}(0), \beta(y))] d y-\mathcal{W}(t) .
$$

We call $Q(t)$ excess heat (or heat in the case of constant profile of temperature), and (5.30) express the first principle of thermodynamics for quasistatic transformations.

Notice that from (5.27), $Q_{N}(t)$ is the time integral of a highly fluctuating random quantity. It is only after the particular space-time scaling that the this quantity converges, and that the limit is a deterministic function of time. In this sense this result is different from similar identifications of heat as a stochastic flux of energy done in the so-called "Stochastic Thermodynamics" (cf. [15] for example). On the other hand in thermodynamics heat is defined as the total flux of energy between the system and the thermal bath, resulting from the complete transformation from one stationary state to another, while it does not attempt to describe the instantaneous flux of energy. But in a quasi-static transformations at each time $t$ a new stationary state is reached.

5.1.2. Free energy and Clausius identity. Define

$$
\widetilde{\mathcal{F}}(t)=\int_{0}^{1} \mathcal{F}(r(y, t), \beta(y)) d x
$$

A straightforward calculation gives

$$
\widetilde{\mathcal{F}}(t)-\widetilde{\mathcal{F}}(0)=\int_{0}^{t} d s \int_{0}^{1} d y \bar{\tau}(s) \partial_{s} r(y, s)=\int_{0}^{t} \bar{\tau}(s) d \mathcal{L}(s)=\mathcal{W}(t)
$$

i.e. Clausius equality for the free energy.

Equivalently, by using the thermodynamic relation $\mathcal{F}=u-\beta^{-1} S$ from (5.16), we have

$$
\int_{0}^{1} d y \beta^{-1}(y)(S(r(y, t), u(y, t))-S(r(y, 0), u(y, 0)))=Q(t)
$$


In the case of constant temperature profile, this reduce to the expected thermodynamic relation $\dot{S}=\beta \dot{Q}$ for quasistatic isothermal thermodynamic trasformations.

\section{REFERENCES}

[1] Bertini L., Gabrielli D., Jona-Lasinio G., and Landim C. Clausius inequality and optimality of quasistatic transformations for nonequilibrium stationary states. Physical review letters, 110(2):020601 (2013).

[2] Bertini L., Gabrielli D., Jona-Lasinio G., and Landim C. Thermodynamic transformations of nonequilibrium states. Journal of Statistical Physics, 149, 773-802 (2012).

[3] Callen H.B., Thermodynamics and introduction to thermostatistics, John Wyles \& sons, New York 1985.

[4] De Masi A., Ferrari P. A. A remark on the hydrodynamics of the zero range process. Journal of Statistical Physics, 36, 81-87 (1984).

[5] De Masi A., Presutti E., Tsagkarogiannis D., and Vares M.E.. Truncated correlations in the stirring process with births and deaths, Electronic Journal of Probability 17 1-35 (2012)

[6] De Masi A., Presutti E., Mathematical methods for hydrodynamic limits , Lecture Notes in Mathematics. 1501. Berlin: Springer-Verlag, 1991

[7] Eyink, G., Lebowitz J.L., Spohn H.. Hydrodynamics of Stationary States for Some Stochastic Lattice Models, Comm.Math.Phys, 132, 253-283 (1990).

[8] Galves A., Kipnis C., Marchioro C. and Presutti E. Non equilibrium measures which exhibt a temperature gradient: study of a model. Commun. Math. Phys., 819, 127-1474 (1981).

[9] Guo M.Z. , Papanicolaou G. C., and Varadhan S.R. Nonlinear diffusion limit for a system with nearest neighbor interactions. Commun. Math. Phys. 118, 31-59 (1988)

[10] Kipnis C., Landim C.. Scaling Limits of Interacting Particle Systems, Grundlehren der mathematischen Wissenschaften 320, Springer-Verlag, Berlin, New York, (1999).

[11] Kipnis C., Olla S., and Varadhan S.R.. Hydrodynamics and large deviation for simple exclusion processes, Commun. Pure Appl. Math. 42 115137(1989).

[12] Landim C., Milanés A. and Olla S.. Stationary and non-equilibrium fluctuations in boundary driven exclusion process Markov Proces. Related Fields Vol. 14, 165-184 (2008).

[13] Letizia V., and Olla S.. Non-equilibrium isothermal transformations in a temperature gradient from a macroscopic dynamics, arXiv:1505.05002. (2015)

[14] Olla S.Microscopic derivation of an isothermal thermodynamic transformation, In From Particle Systems to Partial Differential Equations, pages 225-238. Springer, 2014.

[15] Seifert U., Stochastic thermodynamics, Lecture Notes: Soft Matter. From Synthetic to Biological Materials. 39th IFF Spring School, Institut of Solid State Research, Research Centre Jülich (2008).

[16] Spohn H. Long range correlations for stochastic lattice gases in a nonequilibrium steady state, J. Physica A: Math.Gen. 16, 4275-4291 (1983). 
Anna de Masi, Università Dell'Aquila,, 67100 L'Aquila, Italy

E-mail address: demasi@univaq.it

Stefano Olla, CEREMAde, UMR CNRS 7534, Université Paris-Dauphine, 75775 PARIS-CEdex 16, France

E-mail address: olla@ceremade.dauphine.fr 